\title{
L1157-B1, a factory of complex organic molecules in a Solar-type star forming region
}

\author{
Bertrand Lefloch ${ }^{1 \star}$, C. Ceccarelli ${ }^{1}$, C. Codella ${ }^{2}$, C. Favre ${ }^{2,1}$, L. Podio $^{2}$, \\ C. Vastel ${ }^{3}$, S. Viti ${ }^{4}$, R. Bachiller ${ }^{5}$ \\ ${ }^{1}$ Univ. Grenoble Alpes, CNRS, IPAG, 38000 Grenoble, France \\ ${ }^{2}$ INAF, Osservatorio Astrofisico di Arcetri, Largo Enrico Fermi 5, I-50125 Firenze, Italy \\ ${ }^{3}$ Université de Toulouse, UPS-OMP, IRAP, Toulouse, France \\ ${ }^{4}$ Department of Physics and Astronomy, UCL, Gower St., London, WC1E 6BT, UK \\ ${ }^{5}$ IGN Observatorio Astronómico Nacional, Apartado 1143, 28800 Alcalá de Henares, Spain
}

Accepted 2017 April 3. Received 2017 April 1; in original form 2017 February 18

\begin{abstract}
We report on a systematic search for oxygen-bearing Complex Organic Molecules (COMs) in the Solar-like protostellar shock region L1157-B1, as part of the IRAM Large Program "Astrochemical Surveys At IRAM" (ASAI). Several COMs are unambiguously detected, some for the first time, such as ketene $\mathrm{H}_{2} \mathrm{CCO}$, dimethyl ether $\left(\mathrm{CH}_{3} \mathrm{OCH}_{3}\right)$ and glycolaldehyde $\left(\mathrm{HCOCH}_{2} \mathrm{OH}\right)$, and others firmly confirmed, such as formic acid $(\mathrm{HCOOH})$ and ethanol $\left(\mathrm{C}_{2} \mathrm{H}_{5} \mathrm{OH}\right)$. Thanks to the high sensitivity of the observations and full coverage of the 1,2 and $3 \mathrm{~mm}$ wavelength bands, we detected numerous ( 10-125) lines from each of the detected species. Based on a simple rotational diagram analysis, we derive the excitation conditions and the column densities of the detected COMs. Combining our new results with those previously obtained towards other protostellar objects, we found a good correlation between ethanol, methanol and glycolaldehyde. We discuss the implications of these results on the possible formation routes of ethanol and glycolaldehyde.
\end{abstract}

Key words: physical data and processes: astrochemistry - ISM: jets and outflowsmolecules-abundances - Stars: formation

\section{INTRODUCTION}

There is now substantial observational evidence that protostellar shocks can significantly alter the chemical composition of the medium in which they propagate. Thanks to its remarkably bright molecular line emission, the region L1157-B1 (d=250 pc; Looney et al. 2007) has been the privileged target of many observational studies to investigate the physical and chemical properties of protostellar shocks. Gueth et al. (1996) showed that the L1157-B1 shock was caused by the high-velocity, precessing jet driven by the low luminosity Class 0 source L1157-mm $\left(\mathrm{L} \sim 4 L_{\odot}\right)$. Following the pioneering study of Bachiller \& Perez-Gutierrez (1997), Arce et al. (2008) reported the detection of a few COMs: methyl formate $\left(\mathrm{HCOOCH}_{3}\right)$, methyl cyanide $\left(\mathrm{CH}_{3} \mathrm{CN}\right)$, ethanol $\left(\mathrm{C}_{2} \mathrm{H}_{5} \mathrm{OH}\right)$, and formic acid $(\mathrm{HCOOH})$.

Since then, individual studies have reported the presence of various organic species in that region (e.g. Codella et al. 2010). Codella et al. (2009) proved unambiguously that
$\mathrm{CH}_{3} \mathrm{CN}$ was associated with the shock itself. More recently, Mendoza et al. (2014) have reported the detection of formamide $\mathrm{NH}_{2} \mathrm{CHO}$, with an abundance ranging among the highest values ever reported in molecular clouds (Bisschop et al. 2007; Lopez-Sepulcre et al. 2015). This detection raises the question of the molecular complexity which is ultimately reached in protostellar shocks and the nature of the involved chemical processes, either in the gas phase or at the surface of dust grains.

L1157-B1 was observed as part of ASAl1 with the IRAM $30 \mathrm{~m}$ telescope to investigate the feedback of shocks on the molecular gas. In this Letter, we present and discuss the results of our search for Oxygen-bearing COMs. From a highly sensitive, unbiased spectral line survey of the $72-350$ $\mathrm{GHz}$ band, we have obtained a comprehensive census of the COMs content in L1157-B1. The detected lines span a wide range of excitation, with $E_{u p}$ ranging from about $5 \mathrm{~K}$ up to 40-180 K, and spontaneous emission coefficients $A_{i j}$, from 
$10^{-6} \mathrm{~s}^{-1}$ to a few $10^{-4} \mathrm{~s}^{-1}$, depending on the species. This has allowed us to determine with unprecedented accuracy the excitation conditions and abundances of these species in L1157-B1.

\section{OBSERVATIONS}

The observations of L1157-B1 were carried out with the IRAM-30m telescope at Pico Veleta (Spain), during several runs in 2011 and 2016, and covered the spectral bands $72-$ $116 \mathrm{GHz}, 128-173 \mathrm{GHz}, 200-260 \mathrm{GHz}$, and $260-350 \mathrm{GHz}$. The observed position of L1157-B1 is $\alpha_{J 2000}=20^{\mathrm{h}} 39^{\mathrm{m}} 10{ }^{\mathrm{s}} 2$, $\delta_{J 2000}=+68^{\circ} 01^{\prime} 10^{\prime \prime}$. The survey was carried out using the broad-band EMIR receivers. Fast Fourier Transform Spectrometers were connected to the EMIR receivers, providing a spectral resolution of $195 \mathrm{kHz}$. The high-frequency part band 260-350 GHz was observed with the WILMA autocorrelator, at $2 \mathrm{MHz}$ resolution. The final kinematic resolution of the FTS data was degraded to $1 \mathrm{~km} \mathrm{~s}^{-1}$. A description of the observing procedure and data acquisition will be presented more extensively in the article presenting the ASAI survey (Lefloch in prep).

The data reduction was performed using the GILDAS/CLASS90 packag $\mathrm{I}^{2}$. The line intensities are expressed in units of antenna temperature corrected for atmospheric attenuation and rearward losses $\left(T_{A}^{\star}\right)$. For the ASAI data, the calibration uncertainties are typically 10,15 , and $20 \%$ at $3 \mathrm{~mm}, 2 \mathrm{~mm}$, and $1.3 \mathrm{~mm}$, respectively (Lefloch in prep). For subsequent analysis, fluxes were expressed in main beam temperature units $\left(T_{m b}\right)$. The telescope and receiver parameters (main-beam efficiency Beff, forward efficiency Feff, Half Power beam Width HPBW) were taken from the IRAM webpage 3 . The rms noise achieved is typically $1 \mathrm{mK}, 3-5 \mathrm{mK}$ and $2-5 \mathrm{mK}\left(T_{A}^{*}\right)$ in a velocity interval of $1 \mathrm{~km} \mathrm{~s}^{-1}$ range at $3 \mathrm{~mm}, 2 \mathrm{~mm}$, and $1.3 \mathrm{~mm}$, respectively.

\section{RESULTS AND DISCUSSION}

\subsection{COMs in L1157-B1}

We performed a systematic search from the list of Oxygen-bearing COMs identified in the sun-like protostar IRAS16293-2422 (Jaber et al 2014). We used the CASSIS software (Vastel et al. 2015) for the line identification, using the CDMS and JPL databases. We detected nine Oxygen-bearing COMs: methanol $\left(\mathrm{CH}_{3} \mathrm{OH}\right)$, ketene $\left(\mathrm{H}_{2} \mathrm{CCO}\right)$, ethanol $\left(\mathrm{C}_{2} \mathrm{H}_{5} \mathrm{OH}\right)$, acetaldehyde $\left(\mathrm{CH}_{3} \mathrm{CHO}\right)$, methyl formate $\left(\mathrm{HCOOCH}_{3}\right)$, glycolaldehyde $\left(\mathrm{HCOCH}_{2} \mathrm{OH}\right)$, dimethyl ether $\left(\mathrm{CH}_{3} \mathrm{OCH}_{3}\right)$, formic acid $(\mathrm{HCOOH})$, and formamide $\left(\mathrm{NH}_{2} \mathrm{CHO}\right)$. We also detected the rare isotopologues $\left({ }^{13} \mathrm{C},{ }^{18} \mathrm{O}\right)$ of methanol. Dimethyl ether, glycolaldehyde and ketene are detected for the first time in a protostellar shock. In addition, two other species, whose identification was based on one or two lines (Arce et al. 2008), are now firmly detected: ethanol and

\footnotetext{
2 http://www.iram.fr/IRAMFR/GILDAS/

3 http://www.iram.es/IRAMES/mainWiki/Iram30mEfficiencies
}

formic acid. The detection of formamide was previously reported and discussed by Mendoza et al. (2014). Our results are summarized in Table 1

For each detected species, we could identify from 14 transitions (glycolaldehyde) up to more than 100 transitions (methanol). A montage of illustrative transitions from each species (but methanol) is displayed in Fig. 1. Line intensities are weak, typically ranging between 10 and $20 \mathrm{mK}\left(\mathrm{T}_{\mathrm{A}}^{*}\right)$, with the exception of acetaldehyde, whose transitions display higher $A_{i j}$, and methanol. All the lines are blueshifted with respect to the cloud ambient velocity $v_{l s r}=+2.6 \mathrm{~km} \mathrm{~s}^{-1}$ (the peak intensity lies at $+0 \mathrm{~km} \mathrm{~s}^{-1}$ ) and display typical linewidths of $\sim 4$ to $7 \mathrm{~km} \mathrm{~s}^{-1}$ (FWHM). This supports evidence for emission from the shocked material of L1157-B1 and not from the ambient molecular cloud. Recently, using the PdBI at 2.'5 resolution, Codella et al. (2015) showed that the emission of $\mathrm{CH}_{3} \mathrm{CHO}$ is associated with the outflow cavity of L1157-B1. The similarity of the line profiles (see Fig. 1) suggests that this is probably the case for all the COMs reported here. These detections are all the more remarkable as we did not find any evidence of COM emission towards L1157-mm itself, the protostar at the origin of the outflow phenomenon and its associated shocks (Tafalla et al. 2015; Podio et al. 2016)

\subsection{Molecular abundances}

Given the large number $(\geqslant 10)$ of detected lines for each species, our new observations allow us not only to firmly establish the presence but also to give reliable estimates of the relative abundances of the COMs detected in L1157-B1. With this goal in mind, we obtained their excitation conditions and column density carrying out a simple rotational diagram analysis. We computed the molecules abundances with respect to $\mathrm{H}_{2}$ by using the whole $\mathrm{CO}$ column density in the B1 direction, as estimated by Lefloch et al. (2012) $\mathrm{N}(\mathrm{CO})=2 \times 10^{17} \mathrm{~cm}^{-2}$ and assuming a standard relative $\mathrm{CO}$ abundance $\mathrm{X}(\mathrm{CO})=\mathrm{N}(\mathrm{CO}) / \mathrm{N}\left(\mathrm{H}_{2}\right)=10^{-4}$. The obtained temperatures, column densities, and abundances are summarized in Table 1

The rotational diagrams of the newly detected COMs are shown in Fig. 2. For all the detected species, the line emission can be reasonably well fit by one single gas component whose rotational temperature is around 20-30 K (Tab. 1). Glycolaldehyde has a slightly larger rotational temperature $(\sim 50 \mathrm{~K})$ but also a larger error bar $(\sim 10 \mathrm{~K})$. Considering only the lines observed with Eup $\leqslant 55 \mathrm{~K}$, a lower rotational temperature $(31.1 \pm 5.3 \mathrm{~K})$ would provide a very good fit.

Column densities vary from $\sim 2 \times 10^{12} \mathrm{~cm}^{-2}$ (formamide) to $\sim 1 \times 10^{15} \mathrm{~cm}^{-2}$ (methanol). Incidentally, the column density of the other COMs shows a relatively small range of variation, from $\sim 2$ to $\sim 5 \times 10^{13} \mathrm{~cm}^{-2}$, namely less than a factor of three. In other words, with the exception of formamide, which is significantly less abundant, the other detected COMs have abundances a factor 20 to 50 lower than methanol.

We searched for acetic acid $\left(\mathrm{CH}_{3} \mathrm{COOH}\right)$, the other isomer of glycolaldehyde and methyl formate, using the frequencies given in Ilyushin et al. (2008). We failed to detect it. We could place an upper limit on its abundance, adopting $T_{\text {rot }}$ similar to that of methyl formate $(20 \mathrm{~K})$ and glycolalde- 
Table 1. Oxygen-bearing Complex Organic Molecules detected with ASAI towards the protostellar shock L1157-B1: methanol $(\mathrm{CH} 3 \mathrm{OH})$, ketene $\left(\mathrm{H}_{2} \mathrm{CCO}\right)$, ethanol $\left(\mathrm{C}_{2} \mathrm{H}_{5} \mathrm{OH}\right)$, acetaldehyde $\left(\mathrm{CH}_{3} \mathrm{CHO}\right)$, methyl formate $\left(\mathrm{HCOOCH}_{3}\right)$, glycolaldehyde $\left(\mathrm{HCOCH}_{2} \mathrm{OH}\right)$, dimethyl ether $\left(\mathrm{CH}_{3} \mathrm{OCH}_{3}\right)$, formic acid $(\mathrm{HCOOH})$, and formamide $\left(\mathrm{NH}_{2} \mathrm{CHO}\right)$. For each molecular species, we indicate the database and the TAG used, the range of $E_{u p}$ and $A_{i j}$ spanned by the transitions detected, the number of detected lines, the excitation temperature and the column density obtained from a simple rotational diagram analysis, and the derived abundance with respect to $\mathrm{H}_{2}$. We adopt the convention $a(b)=a \times 10^{b}$.

\begin{tabular}{lrrrrrrrr}
\hline Species & Database & TAG & Lines & $E_{u p}(\mathrm{~K})$ & $A_{i j}\left(\mathrm{~s}^{-1}\right)$ & $T_{\text {rot }}(\mathrm{K})$ & $\mathrm{N}\left(\mathrm{cm}^{-2}\right)$ & $\mathrm{X}$ \\
\hline $\mathrm{CH}_{3} \mathrm{OH}$ & JPL & 32003 & 125 & $4.6-177.5$ & $2.0(-6)-2.3(-4)$ & $25.1 \pm 1.5$ & $(10 \pm 1.0)(14)$ & $(5.0 \pm 1.0)(-7)$ \\
$\mathrm{H}_{2} \mathrm{CCO}$ & CDMS & 42501 & 23 & $9.7-105.5$ & $5.0(-6)-2.1(-4)$ & $23.0 \pm 1.1$ & $(1.7 \pm 0.7)(13)$ & $(8.5 \pm 4.3)(-9)$ \\
$\mathrm{C}_{2} \mathrm{H}_{5} \mathrm{OH}$ & JPL & 46004 & 22 & $9.3-74.4$ & $5.1(-6)-8.5(-5)$ & $28.2 \pm 4.5$ & $(4.6 \pm 1.1)(13)$ & $(2.3 \pm 0.8)(-8)$ \\
$\mathrm{CH}_{3} \mathrm{CHO}$ & JPL & 44003 & 78 & $11.8-94.1$ & $1.5(-5)-5.2(-4)$ & $16.6 \pm 0.7$ & $(2.8 \pm 0.4)(13)$ & $(1.4 \pm 0.4)(-8)$ \\
$\mathrm{HCOOCH}_{3}$ & JPL & 60003 & 57 & $20.1-77.7$ & $1.0(-5)-6.5(-5)$ & $20.7 \pm 1.5$ & $(5.4 \pm 0.8)(13)$ & $(2.7 \pm 0.7)(-8)$ \\
$\mathrm{HCOCH}_{2} \mathrm{OH}$ & JPL & 60006 & 14 & $18.8-93.8$ & $4.0(-6)-2.1(-5)$ & $50.4 \pm 10.2$ & $(3.4 \pm 0.7)(13)$ & $(1.7 \pm 0.5)(-8)$ \\
$\mathrm{HCOCH}_{2} \mathrm{OH}^{a}$ & JPL & 60006 & 9 & $18.8-53.2$ & $4.8(-6)-2.1(-5)$ & $31.1 \pm 5.3$ & $(2.1 \pm 0.3)(13)$ & $(1.0 \pm 0.2)(-8)$ \\
$\mathrm{CH}_{3} \mathrm{OCH}_{3}$ & JPL & 46008 & 31 & $6.7-40.4$ & $1.7(-6)-2.1(-5)$ & $13.9 \pm 1.6$ & $(4.9 \pm 0.9)(13)$ & $(2.5 \pm 0.7)(-8)$ \\
$\mathrm{t}-\mathrm{HCOOH}^{b}$ & JPL & 46005 & 38 & $10.8-110.2$ & $5.5(-6)-1.9(-4)$ & $21.2 \pm 1.1$ & $(1.7 \pm 0.2)(13)$ & $(8.5 \pm 1.9)(-9)$ \\
$\mathrm{NH}_{2} \mathrm{CHO}^{b}$ & CDMS & 45512 & 16 & $10.2-32.5$ & $9.2(-6)-2.5(-4)$ & $10.0 \pm 2.0$ & $(3.5 \pm 1.0)(12)$ & $(1.8 \pm 0.7)(-9)$ \\
& CDMS & 45512 & 8 & $60.8-82.9$ & $2.1(-5)-1.0(-3)$ & $34.0 \pm 2.0$ & $(1.7 \pm 0.2)(12)$ & $(8.5 \pm 1.9)(-10)$ \\
\hline
\end{tabular}

${ }^{a}$ Only lines with $E_{u p} \leqslant 55 \mathrm{~K}$ are considered. ${ }^{b}$ Two excitation components are detected (Mendoza et al. 2014).

hyde $(50 \mathrm{~K})$, and the partition function given by Calcutt et al. (in prep). We obtain a $3 \sigma$ upper limit of $5 \times 10^{-9}$ at $20 \mathrm{~K}$ $\left(10^{-8}\right.$ at $\left.50 \mathrm{~K}\right)$ : acetic acid is less abundant by a factor of 3 to 5 with respect to methyl formate.

\subsection{Comparison with hot corinos}

When compared to other Solar-like star forming regions where these COMs were previously detected (IRAS162932422: Jaber et al. 2014, Coutens et al. 2015, Jørgensen et al. 2016; IRAS2A and IRAS4A: Taquet et al. 2015), L1157-B1 appears to be a site were COMs are very efficiently produced. The molecular abundances are higher in the shock than in the hot corinos by a factor of 2 to 10 , depending on the species. The dimethyl ether abundance measured in IRAS16293-2422 is the only exception. We note that only relatively small COMs are detected in the shock region, whereas larger $\mathrm{COMs}$ like e.g. propanone $\left(\mathrm{CH}_{3} \mathrm{COCH}_{3}\right)$, ethylene glycol $\left(\left(\mathrm{CH}_{2} \mathrm{OH}\right)_{2}\right)$ are detected in the inner protostellar environments (e.g. Maury et al. 2014; Jørgensen et al. 2016) but not yet in the shock. More observational data from hot corinos and shock regions are needed to establish whether these results are general and why.

Following Jaber et al. (2014) and Mendoza et al. (2014), we searched for correlations between pairs of COMs detected in L1157-B1. In addition to the already known correlation between methyl formate and dimethyl ether (Jaber et al. 2014), we found bf good correlations between ethanol, methanol and glycolaldehyde (Fig. 3). Although these species have been detected in only three Solar-type hot corinos, the correlation spans two orders of magnitude and it is robust in both cases.

\subsection{Formation routes for ethanol and glycolaldehyde}

The correlations methanol-ethanol and ethanolglycolaldehyde are intriguing and require further investigation. Indeed, in some cases, correlations have provided a precious key to unveil the formation route of some species.
For example, Jaber at al. (2014) found that the methyl formate and dimethyl ether abundances are very tightly correlated, with an abundance ratio close to unity. This fact led Balucani et al. (2015) to propose a new formation route methyl formate from dimethyl ether in the gas phase. On the contrary, the tight correlation observed between formamide and isocyanic acid (Lopez-Sepulcre et al. 2015) is still not understood.

Hydrogenation of $\mathrm{CO}$ on the grain surfaces is one of the few methanol formation routes for which there is consensus (e.g. Cuppen et al. 2009; Rimola et al. 2014). The correlations methanol-ethanol and ethanol-glycolaldehyde might then suggest that ethanol and glycolaldehyde are formed on the grain surfaces, as suggested by some experiments. However, theoretical computations have so far been unable to support this hypothesis. Enrique-Romero et al. (2016) have shown that the synthesis of acetaldehyde on amorphous water ices, predicted by the same experiments to occur thanks to the combination of $\mathrm{HCO}$ and $\mathrm{CH}_{3}$, is in fact impossible: such a reaction produces methane and $\mathrm{CO}$, not acetaldehyde. Conversely, simple chemical modelling in L1157-B1 suggests that acetaldehyde may be formed in gas-phase if enough $\mathrm{C}_{2} \mathrm{H}_{5}$ is sputtered off the grain mantles in the shock (Codella et al. 2015).

With these caveats, it is worth investigating whether ethanol and glycolaldehyde could form in the gas phase from species present on the grain surfaces and released in the gas phase by the shock. Both the databases KIDA 4 and UMIST 5 report protonated ethanol $\mathrm{C}_{2} \mathrm{H}_{5} \mathrm{OH}_{2}^{+}$as the precursor of ethanol. It is synthesised in the gas by radiative association between $\mathrm{H}_{3} \mathrm{O}^{+}$and $\mathrm{C}_{2} \mathrm{H}_{4}$, and $\mathrm{H}_{2} \mathrm{O}$ and $\mathrm{C}_{2} \mathrm{H}_{5}^{+}$. Since in the shocked gas of L1157-B1 water is abundant (e.g. Lefloch et al. 2010; Busquet et al. 2014) and the ionisation rate is relatively high $\zeta \simeq 3 \times 10^{-16}$ (Podio et al. 2015), ethanol as a gas-phase product is therefore a viable possibility.

Chemical models by Woods et al. (2012, 2013) do explore possible gas-phase routes for the formation of glyco-

\footnotetext{
${ }^{4}$ http://kida.obs.u-bordeaux1.fr

${ }^{5}$ http://udfa.ajmarkwick.net
} 
Figure 1. Montage of detected transitions from the different O-bearing COMs detected towards L1157-B1. Intensities are expressed in units of $T_{A}^{*}$. The red dashed line marks the ambient cloud velocity $v_{l s r}=+2.6 \mathrm{~km} \mathrm{~s}^{-1}$. Predicted line intensities in the LTE regime and based on our rotational diagram analysis are shown in red.

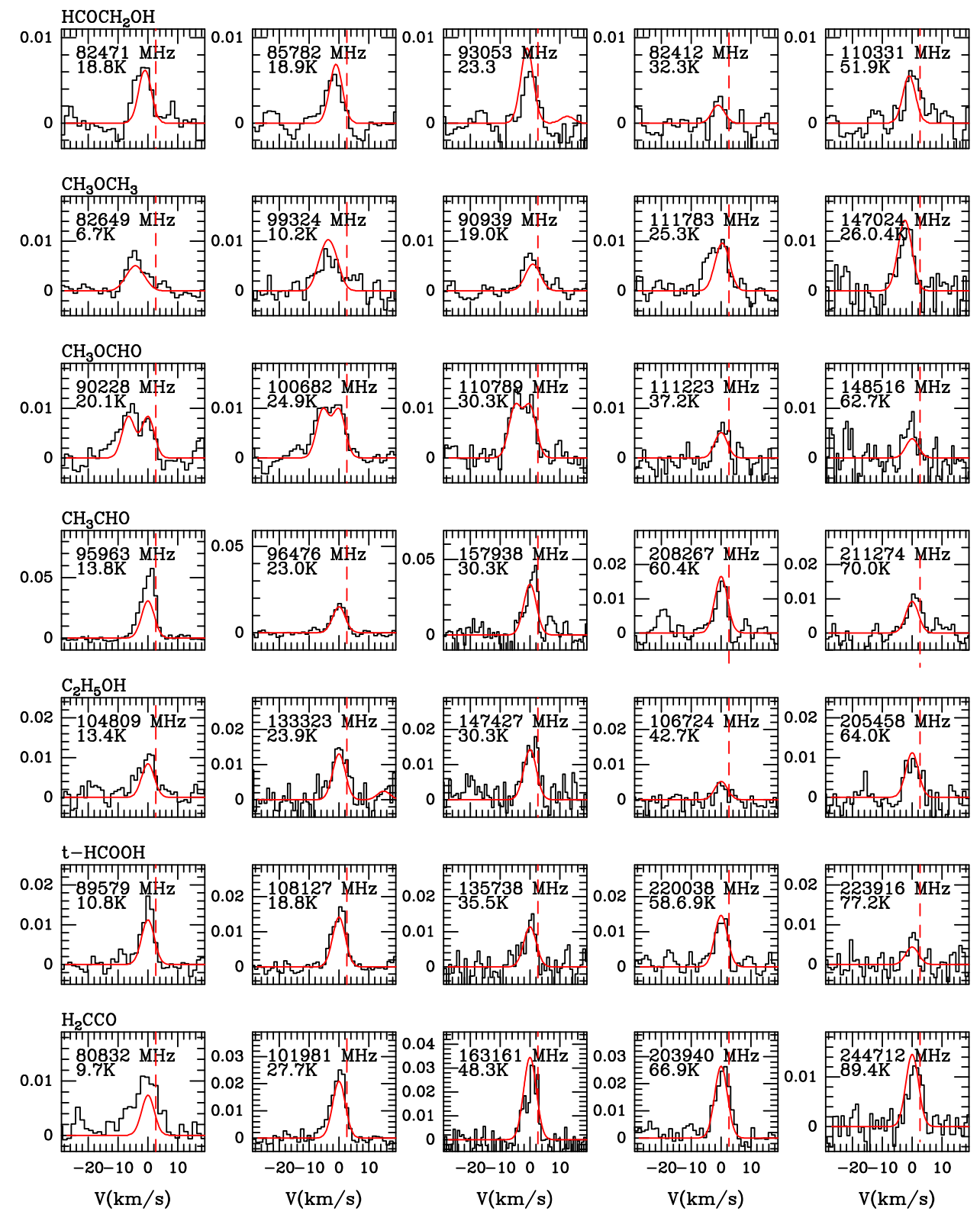

laldehyde. In particular, Woods et al. (2012) include in their models the gas-phase route proposed in Halfen et al. (2006). The authors conclude that the predicted routes are inefficient, due to the lack of available reactants in the gas phase (e.g. formaldehyde). As a consequence of the efficient sputtering of molecular species (e.g. $\mathrm{H}_{2} \mathrm{CO}$ and $\mathrm{CH}_{3} \mathrm{OH}$ ) from grain mantles and the warm temperatures reached, the conditions in the shocked gas of L1157-B1 are more favourable to glycolaldehyde formation. It would be worth exploring the efficiency of these gas phase routes under such conditions.

\section{CONCLUSION}

We have led a systematic search for COMs in the protostellar shock region L1157-B1 as part of the ASAI Large Program. The unprecedented sensitivity of the spectral survey has allowed us to detect nine molecular species, among which $\mathrm{H}_{2} \mathrm{CCO}, \mathrm{CH}_{3} \mathrm{OCH}_{3}$ and $\mathrm{HCOCH}_{2} \mathrm{OH}$ for the first time in a protostellar shock. Also, we confirm the presence of $\mathrm{HCOOH}, \mathrm{HCOOCH}_{3}$ and $\mathrm{C}_{2} \mathrm{H}_{5} \mathrm{OH}$, whose previous tentative detections were based on very few lines. Excitation temperatures and molecular abundances were obtained from a rotational diagram analysis. COMs appear to be more abundant in the shock L1157-B1 than in typical solar-type hot corinos, by a factor of $2-10$, depending on the species. Comparison of COMs abundances in L1157-B1 and hot corinos 

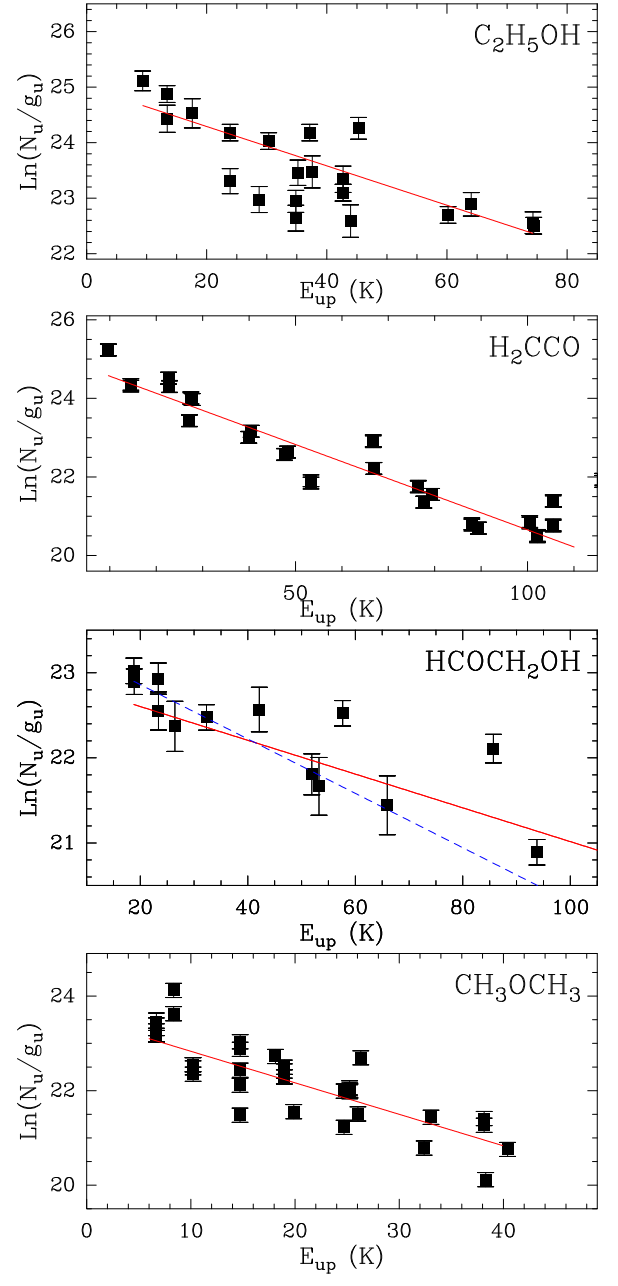

Figure 2. Rotational diagrams of $\mathrm{COMs}$ detected towards L1157-B1. From top to bottom: $\mathrm{C}_{2} \mathrm{H}_{5} \mathrm{OH}, \mathrm{H}_{2} \mathrm{CCO}, \mathrm{HCOCH}_{2} \mathrm{OH}$ and $\mathrm{CH}_{3} \mathrm{OCH}_{3}$. The best fit is drawn by the red line. For $\mathrm{HCOCH}_{2} \mathrm{OH}$, the fit to the transitions with $E_{u p} \leqslant 55 \mathrm{~K}$ is drawn by the blue dashed line.

shows new correlations between ethanol and methanol, and ethanol and glycolaldehyde. Such correlations could be related to the formation route of these molecules, which remain to be established.

\section{ACKNOWLEDGEMENTS}

IRAM is supported by INSU/CNRS (France), MPG (Germany) and IGN (Spain). This work was supported by the CNRS program "Physique et Chimie du Milieu Interstellaire" (PCMI) and by a grant from LabeX Osug@2020 (Investissements d'avenir - ANR10LABX56). C. Favre acknowledges funding from the French CNES agency.

\section{REFERENCES}

Arce,H.G., Santiago-García,J., Jørgensen,J.K., et al., 2008, ApJ, 681, L21

Bachiller, R. \& Pérez Gutiérrez, M., 1997, ApJ, 487, L93
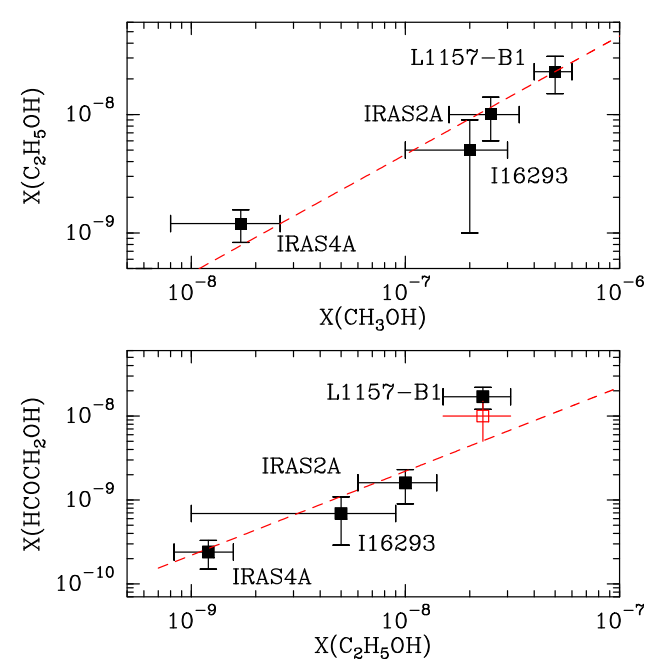

Figure 3. (top) Ethanol abundance as a function of methanol abundance in Hot Corino sources and L1157-B1; the relation $\mathrm{X}\left(\mathrm{C}_{2} \mathrm{H}_{5} \mathrm{OH}\right)=0.046 \mathrm{X}\left(\mathrm{CH}_{3} \mathrm{OH}\right)$ is marked by the red dashed line. (bottom) Same for glycolaldehyde and ethanol. We have plot the Glycolaldehyde abundance estimated from lines with $E_{u p} \leqslant 55 \mathrm{~K}$ (red empty square). The relation $\mathrm{X}\left(\mathrm{HCOCH}_{2} \mathrm{OH}\right)=$ $0.22 \mathrm{X}\left(\mathrm{C}_{2} \mathrm{H}_{5} \mathrm{OH}\right)$ is marked by the red dashed line.

Bisschop, S., E., Jørgensen J. K., van Dishoeck E.F., de Wachter, E.B.M., 2007, A\&A, 465, 913

Busquet,G.,Lefloch,B.,Benedettini,M.,et al.,2014,A\&A, 561, 120 Codella,C.,Lefloch,B.,Ceccarelli,C.,et al., 2010, A\&A, 518, L112

Codella,C.,Fontani,F.,Ceccarelli,C.,et al.,2015,MNRAS, 449, L11 Coutens, A., Persson, M. V., Jørgensen, J. K., et al., 2015, A\&A, 576,5

Cuppen, H.M., van Dishoeck, E. F., Herbst, E., Tielens, A. G. G. M., 2009, A\&A, 508, 275

Enrique-Romero, J., Rimola, A., Ceccarelli, C., Balucani, N., 2016, MNRAS, 459, L6

Gueth, F., Guilloteau, S., Bachiller, R., 1996, A\&A, 307, 891

Ilyushin, V., Kleiner, I., Lovas, F.J., Journal of Physical and Chemical Reference Data, 37, 97

Jaber,A., Ceccarelli,C., Kahane,C., Caux,E., 2014, ApJ, 791, 29

Jalbout,A.F., Abrell, L., Adamowicz, L., et al., 2007, Astrobiology, $7(3), 433$

Jørgensen, J., van der Wiel, M. H. D., Coutens, A., et al., 2016, A\&A, 595, 117

Lefloch, B., Cabrit, S., Codella, C., et al., 2010, A\&A, 518, L113

Lefloch, B., Cabrit, S., Busquet, G., et al., 2012, ApJ, 757, L25

Looney, L. W., Tobin, J. J., \& Kwon, W. 2007, ApJ, 670, L131

Lopez-Sepulcre, A., Jaber, A.A., Mendoza, E., et al., 2015, MNRAS, 449, 2438

Mendoza, E., Lefloch, B., Lopez-Sepulcre, A., et al., 2014, MNRAS, 445, 151

Podio, L., Lefloch B., Ceccarelli C., et al., 2014, A\&A, 565, 64

Podio, L., Codella, C., Gueth, F., et al., 2016, A\&A, 593, L4

Rimola, A., Taquet, V., Ugliengo, P, et al., 2014, A\&A, 572, 70

Tafalla, M., Bachiller, R., Lefloch, B., et al., 2015, A\&A, 573, L2

Taquet, V., Lopez-Sepulcre, A., Ceccarelli, C., et al., 2015, ApJ, 804,81

Vastel, C., Bottinelli,S., Caux, E., et al., 2015, Proceedings of the Annual meeting of the French Society of Astron. and Astrophysics. Eds.: F. Martins, S. Boissier, V. Buat, L. Cambrésy, P. Petit, pp.313-316

Woods,P.M., Kelly,G., Viti,S., et al., 2012, ApJ, 750, 19

Woods,P.M., Slater, B., Raza, Z., et al., 2013, ApJ, 777, 90 